Monatsh. Math. 129, 303-319 (2000)

Monatshefte für

Mathematik

(C) Springer-Verlag 2000

Printed in Austria

\title{
Smooth Stable Planes and the Moduli Spaces of Locally Compact Translation Planes
}

\author{
By \\ Richard Bödi ${ }^{1}$, Stefan Immervoll ${ }^{1}$, and Harald Löwe ${ }^{2}$ \\ ${ }^{1}$ Universität Tübingen, Germany \\ ${ }^{2}$ Technische Universität Braunschweig, Germany
}

(Received 15 April 1999; in revised form 22 October 1999)

\begin{abstract}
Smooth stable planes have been introduced in [3]. At every point $p$ of a smooth stable plane $S=(P, \mathscr{L}, \mathscr{F})$ the tangent spaces of the lines through $p$ form a compact spread (see the definition in Section 2) on the tangent space $\mathrm{T}_{p} P$ thus defining a locally compact topological affine translation plane $\mathscr{A}_{p}$. We introduce the moduli space $\mathrm{J}_{l}\left(\mathbb{R}^{2 l}\right)$ of isomorphism classes of compact spreads, $l \in\{1,2,4,8\}$. We show that for $l>1$ the topology of $\mathrm{J}_{l}\left(\mathbb{R}^{2 l}\right)$ is not $\mathrm{T}_{1}$ by constructing a sequence of non-classical spreads in $\mathbb{F}^{2}$ that converges to the classical spread in $\mathbb{F}^{2}$, where $\mathbb{F} \in\{\mathbb{C}, \mathbb{H}, \mathbb{O}\}$. Moreover, we prove that the isomorphism type of $\mathscr{A}_{p}$ varies continuously with the point $p$. Finally, we give examples of smooth affine planes which have both classical and non-classical tangent translation planes.
\end{abstract}

1991 Mathematics Subject Classification: 51H25, 51A40

Key words: Stable plane, translation plane, moduli space, smooth planes

Topological geometry was born in 1932 when Kolmogoroff published his paper Zur Begründung der projektiven Geometrie [12]. He was the first who combined incidence structures (projective spaces) and topology. Skornyakov (1954) and Freudenthal (1957) continued Kolmogoroff's ideas. In the late '50s, Salzmann started the systematic investigation of topological plane geometries. An account of this theory is given in the recent book Compact Projective Planes [21].

The four classical examples of compact projective planes, namely the projective planes $\mathscr{P}_{2} \mathbb{K}$ over the locally compact division algebras $\mathbb{K} \in\{\mathbb{R}, \mathbb{C}, \mathbb{H}, \mathbb{O}\}$, are not only topological planes; the set of points and the set of lines are, in fact, smooth manifolds and the geometric operations of join and intersection are not only continuous but even smooth. This leads to the notion of smooth incidence structures. In the present paper we will study smooth stable planes (see the definition in Section 1). These objects were first investigated in [3], [4], and [5]. The fact that every continuous collineation of a smooth stable plane is in fact a smooth collineation (see [4]) implies that the smooth structures (on the set of points and the set of lines) of a smooth stable plane are uniquely determined by the underlying topological stable plane. Among the nice features of smooth stable planes are the so-called tangent translation planes which live on the tangent spaces $\mathrm{T}_{p} P$ of points $p$. We will give a construction of these tangent planes in Section 2. Translation planes in general are uniquely determined by so-called spreads. We will introduce the moduli space $\mathrm{J}_{l}(V)$ 
consisting of isomorphism classes of spreads on a real $2 l$-dimensional vector space $V, l \in\{1,2,4,8\}$. We will prove and make precise the following results:

Theorem A. For $l>1$, the moduli space $J_{l}(V)$ does not satisfy the $T_{1}$ separation axiom.

Theorem B. For every smooth stable plane $\mathscr{S}$ the isomorphism type of the tangent translation plane $\mathscr{A}_{p}$ varies continuously with the point $p$.

Theorem C. There exist smooth affine planes which have non-isomorphic tangent translation planes $\mathscr{A}_{p}$ and $\mathscr{A}_{q}$ at some points $p$ and $q$.

The authors would like to thank the referees for their valuable comments and suggestions which helped to improve this paper considerably.

\section{Basic Notions}

The definition of a smooth stable plane is based on the purely incidence geometric notion of a linear space. A linear space $\mathscr{S}=(P, \mathscr{L}, \mathscr{F})$ consists of sets $P, \mathscr{L}$ and $\mathscr{F}$, where $P$ denotes the set of points, $\mathscr{L}$ is the set of lines and $\mathscr{F} \subseteq P \times \mathscr{L}$ is the set of flags, such that for every pair of distinct points $p, q$ there is exactly one joining line $L=p \vee q \in \mathscr{L}$, i.e. $(p, L),(q, L) \in \mathscr{F}$. If $(p, L) \in \mathscr{F}$, we shall say that $p$ and $L$ are incident, or $p$ lies on $L$, or $L$ passes through $p$. Sometimes it is convenient to identify each line $L$ of a linear space with the set of points that are incident with $L$, i.e. we identify $L$ with the set $\{p \in P \mid(p, L) \in \mathscr{F}\}$. If we look at a line this way, we frequently will call it a point row. Similarly, a pencil $\mathscr{L}_{p}$ of lines is the set of all lines that are incident with a given point $p$.

The map $\vee: P \times P \backslash \operatorname{diag}_{P} \rightarrow \mathscr{L}$ which assigns to each pair of distinct points its joining line is called the join map. The intersection map $\wedge$ is defined dually. Note that in a linear space two lines may not intersect. Hence, for a linear space, the map $\wedge$ need not be defined on the whole set $\mathscr{L} \times \mathscr{L} \backslash \operatorname{diag}_{\mathscr{L}}$.

A stable plane $\mathscr{S}$ is a linear space $(P, \mathscr{L}, \mathscr{F})$ that satisfies the following axioms.

(S1) There are Hausdorff topologies on both $P$ and $\mathscr{L}$ that are neither discrete nor antidiscrete such that the join map $\vee$ and the intersection map $\wedge$ are continuous. Morover, the domain $\mathcal{O}$ of the intersection map is an open subset of $\mathscr{L} \times \mathscr{L}$ (stability axiom).

(S2) The topology on $P$ is locally compact and has positive finite covering dimension. line.

(S3) $\mathscr{S}$ contains four points such that any three of them do not lie on a common

A smooth stable plane $\mathscr{S}$ is a stable plane $(P, \mathscr{L}, \mathscr{F})$ such that $P$ and $\mathscr{L}$ are smooth manifolds and such that the join and intersection maps are smooth on their (respective) domains. A smooth stable plane $\mathscr{A}$ is called a smooth affine plane if it is an affine plane (from the incidence geometric point of view) and if the map which assigns to each line and each point $p$ the parallel line through $p$ is smooth.

We will always consider a smooth stable plane $\mathscr{S}=(P, \mathscr{L}, \mathscr{F})$ with lines of dimension $l$. According to Löwen, [16], or Breitsprecher, [6], we have 
$n=\operatorname{dim} P=2 l$, where the integer $l$ is one of the numbers 1,2,4,8. By [3], (2.6) and (2.14), every point row is a closed submanifold of $P$, every line pencil is a compact submanifold of $\mathscr{L}$, and the flag space $\mathscr{F}$ is a closed submanifold of the product manifold $P \times \mathscr{L}$. The restrictions $\pi_{P}: \mathscr{F} \rightarrow P$ and $\pi_{\mathscr{L}}: \mathscr{F} \rightarrow \mathscr{L}$ of the canonical projections $P \times \mathscr{L} \rightarrow P$ and $P \times \mathscr{L} \rightarrow \mathscr{L}$ to $\mathscr{F}$ are submersions, see [3], end of Section 2.

\section{Tangent Translation Planes and Moduli Spaces of Translation Planes}

Let $(P, \mathscr{L}, \mathscr{F})$ be a smooth stable plane of dimension $n=2 l$. Consider the tangent space $\mathrm{T}_{p} P$ at some point $p \in P$. On $\mathrm{T}_{p} P$ we can define an affine translation plane $\mathscr{A}_{p}$ in the following way. The set of points is just $\mathrm{T}_{p} P$ and the collection $\mathscr{S}_{p}:=\left\{\mathrm{T}_{p} L \mid L \in \mathscr{L}_{p}\right\}$ of $l$-dimensional subspaces serves as the line pencil through the origin $0 \in \mathrm{T}_{p} P$. By [3], we have (SP1) $\bigcup_{K \in \mathscr{L}_{p}} \mathrm{~T}_{p} K=\mathrm{T}_{p} P$ and (SP2) $\mathrm{T}_{p} L \oplus \mathrm{T}_{p} K=\mathrm{T}_{p} P$ for any two distinct lines $K, L \in \mathscr{L}_{p}$. Any family of $l$-dimensional subspaces of a $2 l$-dimensional vector space $V$ that satisfies these two axioms is called a spread in $V$. It is a well-known result that every spread $\mathscr{S}$ in $V$ defines a translation plane $\mathscr{A}$ on the point set $V$ by defining $\mathscr{L}:=\{W+v \mid W \in \mathscr{S}, v \in V\}$ as the set of lines of $\mathscr{A}$. By [3], (3.5), the planes $\mathscr{A}_{p}$ are even topological affine translation planes. In particular, the map $\rho_{p}$ which assigns to every non-zero vector $v$ of $\mathrm{T}_{p} P$ the (unique) tangent space $\mathrm{T}_{p} L$ containing $v$ is a continuous map (with respect to the Grassmann topology). According to Löwen, [17], the plane $\mathscr{A}_{p}$ is a topological affine plane if and only if the spread $\mathscr{S}_{p}$ is compact (and in this case $\mathscr{S}_{p}$ is homeomorphic to the $l$ sphere). We refer to $\mathscr{A}_{p}=\left(\mathrm{T}_{p} P, \mathscr{S}_{p}\right)$ as the tangent translation plane at $p$ and to $\mathscr{S}_{p}$ as the tangent spread at $p$. It is unknown whether or not the tangent translation planes are smooth affine planes in general. However, we will prove that the smooth affine planes which are constructed in the last section do have smooth tangent translation planes. Note that the tangent incidence structure $\mathscr{A}_{L}$ at some line $L$, which is defined dually to $\mathscr{A}_{p}$, need not be an affine plane. While axiom (SP2) remains valid, axiom (SP1) may not be true anymore. Nevertheless, the incidence structure $\mathscr{A}_{L}$ is the dual of a so-called shear plane which is a special kind of a stable plane. In fact, shear planes can be regarded as a generalization of dual translation planes in the category of stable planes, see [3], Section 4, and [14].

Each spread $\mathscr{S}$ of a real $2 l$-dimensional vector space $V$ can be thought of as a subset of the Grassmann manifold $\mathrm{G}_{l}(V)$. Let $\mathrm{K}_{l}(V)$ be the set of all compact subsets of the Grassmannian $\mathrm{G}_{l}(V)$ taken with the Hausdorff topology, induced by the Hausdorff metric. Convergence with respect to this topology can be described as follows: a sequence $\left(K_{n}\right)_{n \in \mathbb{N}}$ of compact subsets of $\mathrm{G}_{l}(V)$ converges to a compact subset $K$ of $\mathrm{G}_{l}(V)$ if and only if every element of $K$ is a limit point of some sequence $\left(x_{n}\right)_{n \in \mathbb{N}}$ with $x_{n} \in K_{n}$ and, on the other hand, every cluster point of such a sequence is contained in $K$. Consider the subspace $S_{l}(V)$ of $\mathrm{K}_{l}(V)$ consisting of all compact spreads on $V$. We take $S_{l}(V)$ with the subspace topology. Since the spread $\mathscr{S}$ completely determines the translation plane $\mathscr{A}$, we can think of $\mathscr{A}$ as an element of the topological space $S_{l}(V)$. Finally, we introduce an equivalence relation on $S_{l}(V)$ by calling two spreads equivalent if and only if they determine isomorphic translation planes. Note that two translation planes $(V, \mathscr{S})$ 
and $\left(V, \mathscr{S}^{\prime}\right)$ are isomorphic if and only if there exists a $\mathbb{R}$-linear regular mapping $\lambda: V \rightarrow V$ which maps $\mathscr{S}$ onto $\mathscr{S}^{\prime}$, see Knarr [11]. Thm. 1.18. The resulting quotient space is denoted by $\mathrm{J}_{l}(V)$, and we topologize $\mathrm{J}_{l}(V)$ by taking the quotient topology.

2.1 Definition. Let $V$ be a $2 l$-dimensional real vector space. The set $\mathrm{J}_{l}(V)$ of isomorphism classes of compact spreads on $V$ is called the moduli space of $2 l$ dimensional translation planes (with point space $V$ ).

Finally, we describe another way to introduce a topology on the moduli space $\mathrm{J}_{l}(V)$. If $\mathscr{S} \in \mathrm{S}_{l}(V)$ is a compact spread, then the lines of the corresponding translation plane $\mathrm{A}_{2}(\mathscr{S})$ are precisely the affine cosets of the elements of $\mathscr{S}$. We follow Löwen, [17], for a description of the topology of $\mathrm{A}_{2}(\mathscr{S})$ : consider $V$ as a hyperplane of $W=V \oplus \mathbb{R}$. Set $P:=\mathrm{G}_{1}(W) \backslash \mathrm{G}_{1}(V)$ and $\mathscr{L}=\mathscr{L}(\mathscr{S}):=$ $\left\{X \in \mathrm{G}_{l+1}(W) \mid X \cap V \in \mathscr{S}\right\}$. We endow both $\mathscr{P}$ and $\mathscr{L}$ with the Grassmannian topology. Then $(P, \mathscr{L})$ is a topological affine translation plane (incidence is inclusion) which is isomorphic to $\mathrm{A}_{2}(\mathscr{S})$.

Let $\mathrm{S}_{l}^{\prime}(P)$ be the family of all subsets $\mathscr{L} \subseteq G_{l+1}(W)$ such that the intersection $\mathscr{L} \cap V:=\{L \cap V \mid L \in \mathscr{L}\}$ is a compact spread on $V$. By our discussion above, $\mathrm{S}_{l}^{\prime}(P)$ is the family of the line sets of all topological affine translation planes with point set $P$. Note that such sets $\mathscr{L}$ are actually contained in $\mathrm{G}_{l+1}(W) \backslash \mathrm{G}_{l+1}(V)$ as closed subsets. We endow $\mathrm{S}_{l}^{\prime}(P)$ with the Hausdorff topology induced by the Hausdorff metric; see Busemann [7], (3.8). Convergence with respect to this topology can be described similarly as above: as sequence $\left(\mathscr{L}_{i}\right)_{i}$ with $\mathscr{L}_{i} \in \mathrm{S}_{l}^{\prime}(P)$ converges to $\mathscr{L} \in \mathrm{S}_{l}^{\prime}(P)$ if and only if every element of $\mathscr{L}$ is the limit of a sequence $\left(L_{i}\right)_{i}$ with $L_{i} \in \mathscr{L}_{i}$ and, on the other hand, every accumulation point $X$ with $X \not \leq V$ of such a sequence is an element of $\mathscr{L}$.

The composition $\pi^{\prime}=\pi \circ \phi$ of the map $\phi: \mathrm{S}_{l}^{\prime}(P) \rightarrow \mathrm{S}_{l}(V): \mathscr{L} \mapsto \mathscr{L} \cap V$ with the quotient map $\pi: \mathrm{S}_{l}(V) \rightarrow \mathrm{J}_{l}(V)$ maps an element $\mathscr{L} \in \mathrm{S}_{l}^{\prime}(P)$ to the isomorphism class of the affine translation plane $(P, \mathscr{L})$. Therefore, the moduli space $\mathrm{J}_{l}(V)$ can also be considered as a quotient space of $\mathrm{S}_{l}^{\prime}(P)$. The next lemma shows that $\phi$ is a homeomorphism. We conclude that $\mathrm{S}_{l}^{\prime}(P)$ and $\mathrm{S}_{l}(V)$ induce the same topology on $\mathrm{J}_{l}(V)$.

2.2 Lemma. The map $\phi$ is a homeomorphism, i.e. the two descriptions of the moduli space agree.

Proof. Clearly, $\phi$ is a bijective map. Since the topologies on $\mathrm{S}_{l}(V)$ and $S_{l}^{\prime}(P)$ are induced by metrics, $\phi$ is a homemorphism if and only if both $\phi$ and $\phi^{-1}$ are sequentially continuous. Let $\left(\mathscr{L}_{i}\right)_{i}$ be a sequence in $\mathrm{S}_{l}^{\prime}(P)$ which converges to $\mathscr{L} \in \mathrm{S}_{l}^{\prime}(P)$. Set $\mathscr{S}=\phi(\mathscr{L})=\mathscr{L} \cap V$ and $\mathscr{S}_{i}=\phi\left(\mathscr{L}_{i}\right)$. We have to prove that $\mathrm{H}-\lim _{i \rightarrow \infty} \mathscr{S}_{i}=\mathscr{S}$, where H-lim denotes the Hausdorff limit. Consider an element $X \in \mathscr{S}$ and choose a one-dimensional subspace $U \in \mathrm{G}_{1}(W) \backslash \mathrm{G}_{1}(V)$. Then $U+X$ is an element of $\mathscr{L}$ and we have $U+X=\lim _{i \rightarrow \infty} L_{i}$ for an appropriate sequence $\left(L_{i}\right)_{i}$ with $L_{i} \in \mathscr{L}_{i}$. Thus, $X=(U+X) \cap V=\lim _{i \rightarrow \infty}\left(L_{i} \cap V\right)$ is the limit of the sequence $\left(X_{i}\right)_{i}$, where $X_{i}=L_{i} \cap U \in \mathscr{S}_{i}$. Similarly, one may show that every accumulation point of a sequence $\left(X_{i}\right)_{i}$ with $X_{i} \in \mathscr{S}_{i}$ is an element of $\mathscr{S}$; just observe that $X_{i}+U$ is an element of $\mathscr{L}_{i}$ and that the sequence $\left(X_{i}+U\right)_{i}$ has 
$X+U$ as an accumulation point. This shows that $\mathrm{H}-\lim _{i \rightarrow \infty} \mathscr{S}_{i}=\mathscr{S}$. Thus $\phi$ is (sequentially) continuous. With similar arguments we obtain that $\phi^{-1}$ is continuous, too. This finishes the proof.

\section{Proof of Theorem A}

We consider the set $\mathrm{S}_{l}(V)$ of all compact spreads on a $2 l$-dimensional real vector space $V$. Recall that two (compact) spreads define isomorphic translation planes if and only if they can be transformed into each other by a linear automorphism of $V$. Moreover, the linear group $\mathrm{GL}(V)$ acts continuously on the Grassmannian $\mathrm{G}_{l}(V)$. Thus the moduli space $\mathrm{J}_{l}(V)$ can be considered as the orbit space of the natural action of $\mathrm{GL}(V)$ on $\mathrm{S}_{l}(V)$.

We will prove that $J_{l}\left(\mathbb{R}^{2 l}\right)$ does not satisfy the $\mathrm{T}_{1}$ separation axiom for $l>1$ : there exists a sequence of pairwise isomorphic non-classicial spreads converging to the classical spread on $\mathbb{R}^{2 l}$. This will prove Theorem A.

E.N. Kuz'min, [13], analogously introduced the moduli space of real division algebras of a fixed dimension and mentioned without proof that this space is not Hausdorff.

3.1 Example. Let $\mathbb{F} \in\{\mathbb{C}, \mathbb{M}, \mathbb{O}\}$ and let $l$ be the dimension of the real division algebra $\mathbb{F}$. Consider $V=\mathbb{F} \times \mathbb{F}$ as a $2 l$-dimensional real vector space. For every homeomorphism $\rho_{1}:[0, \infty) \rightarrow[0, \infty)$ we may construct a compact spread as follows. Set $S:=\{0\} \times \mathbb{F}$. For each positive real number $r$ and $a \in \mathbb{F}$ with $|a|=1$ let

$$
L_{1}(a, r):=\left\{\left(x, a \cdot\left(r \cdot \operatorname{Re}(x)+\rho_{1}(r) \cdot \operatorname{Pu}(x)\right)\right)^{t} \mid x \in \mathbb{F}\right\},
$$

where $x=\operatorname{Re}(x)+\operatorname{Pu}(x)$ is the decomposition of $x$ into its real and its pure part. Then the family

$$
\mathscr{S}_{1}=\left\{L_{1}(a, r)|r \in \mathbb{R}, r \geqslant 0, a \in \mathbb{F},| a \mid=1\right\} \cup\{S\}
$$

is a compact spread on $V$, i.e. $\mathscr{S}_{1}$ is an element of $\mathrm{S}_{l}(V)$. These spreads where first defined and investigated by Betten [1] (for $\mathbb{F}=\mathbb{C}$ ), Hähl [8] (for $\mathbb{F}=\mathbb{U}$ ), and Hähl [9] (for $\mathbb{F}=\mathbb{O}$ ) and play an important rôle in the classification of locally compact translation planes with large automorphism groups. In particular, if the homeomorphism $\rho_{1}$ is not linear, then the corresponding translation plane is not isomorphic to the classical plane. These planes are coordinatized over so-called generalized mutations of the division algebra $\mathbb{F}$, see [21], 82.21.

We assume that $\rho_{1} \neq \mathrm{id}$ is the identity on $[0,1]$. This implies that the sets $L_{1}(a, r)$ are classical lines (i.e. they are lines of the classical affine plane $\mathscr{A}_{2} \mathbb{F}$ ) if $r \leqslant 1$. Thus, $\mathscr{S}_{1}$ contains an open part of the classical spread. We will blow up this "classical part" by using the linear maps

$$
f_{s}: \mathbb{F} \times \mathbb{F} \rightarrow \mathbb{F} \times \mathbb{F}:(x, y) \mapsto(x, s \cdot y),
$$

where $s$ is a positive real number. Define $\mathscr{S}_{s}:=f_{s}\left(\mathscr{S}_{1}\right)$. Clearly, the family $\mathscr{S}_{s}$ again is a compact spread. Another way to obtain $\mathscr{S}_{s}$ is to replace the homeomorphism $\rho_{1}$ in the above construction by

$$
\rho_{s}:[0, \infty) \rightarrow(0, \infty): r \mapsto \begin{cases}r & \text { if } r \leqslant s \\ s \cdot \rho_{1}\left(\frac{r}{s}\right) & \text { if } r \geqslant s .\end{cases}
$$


We claim that for any sequence $\left(s_{j}\right)_{j}$ converging to $\infty$, the spreads $\mathscr{S}_{s_{j}}$ converge to the classical spread in $\mathbb{F}^{2}$. Let $\left(L_{s_{j}}\left(a_{j}, r_{j}\right)\right)_{j}$ be a sequence that converges to some $l$-dimensional real linear subspace of $\mathbb{F}^{2}$. Since $\left|a_{j}\right|=1$ for any $j \in \mathbb{N}$, the sequence $\left(a_{j}\right)_{j}$ has some cluster point $a$ and we may assume that $\lim _{j \rightarrow \infty} a_{j}=a$. If $r_{j} \leqslant s_{j}$ for almost all $j$, then $L_{s_{j}}\left(a_{j}, r_{j}\right)$ is a classical line for almost all $j$ and hence the limit $L$ of this sequence is classical as well. Thus, by passing to some subsequence, we may suppose that $r_{j}>s_{j}$ for all $j$. Then $\lim _{j \rightarrow \infty} r_{j}=\infty$ and we infer that the sequence $\left(L_{s_{j}}\left(a_{j}, r_{j}\right)\right)_{j}$ has $S$ as its limit. Conversely, every classical line can be written as the limit of such a sequence: for a classical line $L=\{(x, b x) \mid x \in \mathbb{F}\}$ we write $b=a r$ with $|a|=1$ and $r \in \mathbb{R}, r \geqslant 0$. Then, for any sequence $\left(s_{j}\right)$ of real numbers with $s_{j} \geqslant r$ we have $L_{s_{j}}(a, r)=L$. This shows that the Hausdorff limit of $\left(\mathscr{S}_{s_{j}}\right)_{j}$ is the classical spread in $\mathbb{F}^{2}$.

We immediately get the following result, see the discussion before (3.1):

3.2 Corollary. For $l>1$, the moduli space $J_{l}(V)$ is not a $T_{1}$-space.

This result proves Theorem A.

The subgroup $\{A \in \mathrm{GL}(V) \mid A S \in \mathscr{S}$ for all $S \in \mathscr{S}\}$ is called the automorphism group of a spread $\mathscr{S}$ on some vector space $V$. The automorphism group of a compact spread $\mathscr{S}$ is of course a closed subgroup of $\mathrm{GL}(V)$ and thus is a Lie group. In the example given in (3.1) the non-classical spreads $\mathscr{S}_{s}=f_{s}(\mathscr{S})$ converge to the classical spread. Therefore, the dimension of the automorphism group increases when we pass to the limit, cp. [21], introduction to Chapter 8. In fact, this upper semi-continuity holds in general.

3.3 Lemma. Let $\mathscr{A}$ and $\mathscr{B}$ be compact spreads on $\mathbb{R}^{2 l}$ having $\mathrm{A}$ and $\mathrm{B}$ as automorphism groups. Let $\varphi_{i} \in \mathrm{GL}_{2 l} \mathbb{R}$ with $\mathrm{H}-\lim _{i \rightarrow \infty} \varphi_{i}(\mathscr{A})=\mathscr{B}$. Then $\operatorname{dim} A \leqslant \operatorname{dim} B$.

Proof. Let $\mathfrak{a}$ and $\mathfrak{b}$ be the Lie algebras of the Lie groups A and B, respectively. Then the Lie algebra of the automorphism group of the spread $\varphi_{i}(\mathscr{A})$ is just $\mathfrak{a}_{i}:=\varphi_{i} \mathfrak{a} \varphi_{i}^{-1}$. In particular, we have $\operatorname{dima}_{i}=\operatorname{dima}=m$ for any $i \in \mathbb{N}$. Thus, the sequence $\left(\mathfrak{a}_{i}\right)_{i}$ has an accummulation point $\boldsymbol{D}$ in the Grassmannian $\mathrm{G}_{m}\left(\mathfrak{g l}_{2 l} \mathbb{R}\right)$. Of course, the linear subspace $\mathfrak{D}$ is a Lie subalgebra of $\mathfrak{g l}_{2 l} \mathbb{R}$. We claim that the group $\Gamma^{*}:=\langle\exp \mathfrak{D}\rangle$ leaves invariant the spread $\mathscr{B}$ and thus is a subgroup of B. For $R \in \mathscr{A}$ and $\beta \in \operatorname{exp~D}$ near the identity choose sequences $\left(R_{i}\right)_{i}$ with $R_{i} \in \varphi_{i}(\mathscr{A})$ and $\left(\beta_{i}\right)_{i}$ with $\beta_{i} \in \varphi_{i} \mathrm{~A} \varphi_{i}^{-1}$ such that

$$
\lim _{i \rightarrow \infty} R_{i}=R \quad \text { and } \quad \lim _{i \rightarrow \infty} \beta_{i}=\beta .
$$

Then the sequence $\left(\beta_{i}\left(R_{i}\right)\right)_{i}$ converges to $\beta(R)$ in the Grassmannian $\mathrm{G}_{l}\left(\mathbb{R}^{2 l}\right)$. Since the limit of this sequence is contained in the spread $\mathscr{B}$, this proves the lemma.

Retain the notation of the previous lemma. We have seen that every accumulation point $\Gamma^{*}$ of the sequence $\Gamma_{i}=\varphi_{i} \mathrm{~A} \varphi_{i}-1$ (or, more precisely, the group corresponding to an accumulation point of the Lie algebras $\mathfrak{a}_{i}=\varphi_{i} \mathfrak{a} \varphi_{i}^{-1}$ ) has the same dimension as $A$. However, the isomorphism types of $A$ and $\Gamma^{*}$ may differ extremely, as our next example shows. 
3.4 Theorem. There is a sequence of compact spreads $\left(\mathscr{S}_{i}\right)_{i}$ on $\mathbb{R}^{4}$ with the following properties:

(1) For each $i$, the spread $\left(\mathscr{S}_{i}\right)_{i}$ is isomorphic to the singular spread $\mathscr{S}$ with irreducible $\mathrm{SL}_{2} \mathbb{R}$-action; see Betten [2] or [21, 73.17, 73.19 and 73.20]. In particular, the automorphism group $\Gamma_{i}$ of $\mathscr{S}_{i}$ is isomorphic to $\mathrm{GL}_{2} \mathbb{R}$.

(2) The spreads $\left(\mathscr{S}_{i}\right)_{i}$ converge to the classical complex spread $\mathscr{S}_{\mathbb{C}}$ in the Hausdorff sense.

(3) The groups $\Gamma_{i}$ converge to a solvable group $\Gamma^{*} \cong \mathbb{R} \times\left(\mathbb{R}^{2} \rtimes \mathrm{SO}_{2} \mathbb{R}\right)$. Here, convergence is defined as convergence of the Lie algebras in the Grassmannian $\mathrm{G}_{4}\left(\mathfrak{g l}_{4} \mathbb{R}\right)$.

Proof. (a) Let $V=\mathbb{R}^{4}$ and let $S$ be the subspace $S=\{0\} \times \mathbb{R}^{2}$ of $V$. For $a, b, c$, $d \in \mathbb{R}$ we define subspaces

$$
\left|\begin{array}{ll}
a & b \\
c & d
\end{array}\right|=\left\{(x, y, a x+b y, c x+d y)^{t} \mid x, y \in \mathbb{R}\right\} \subseteq V
$$

in their Grassmann coordinates with respect to $W=\mathbb{R}^{2} \times\{0\}$ and $S$. The set $\mathscr{S}=\mathscr{S}_{1} \cup \mathscr{S}_{2} \cup \mathscr{S}_{3}$, where

$$
\begin{aligned}
& \mathscr{S}_{1}=\{S\} \cup\left\{\left|\begin{array}{ll}
-3 b^{2} & 2 b \\
-2 b^{3} & b^{2}
\end{array}\right| \mid b \in \mathbb{R}\right\}, \\
& \mathscr{S}_{2}=\left\{\left|\begin{array}{cc}
-3 b^{2}+d^{2} & 2 b \\
-2 b^{3}-2 b d^{2} & b^{2}+d^{2}
\end{array}\right| \mid b, d \in \mathbb{R}, d>0\right\}, \\
& \mathscr{S}_{3}=\left\{\left|\begin{array}{cc}
-3 b^{2}-3 d^{2} & 2 b \\
-2 b^{3}-2 b d^{2} & b^{2}-d^{2} / 3
\end{array}\right| \mid b, d \in \mathbb{R}, d>0\right\},
\end{aligned}
$$

is a compact spread ([12], 73.19 and 73.20). Moreover, the image $\lceil$ of the irreducible representation $\omega: \mathrm{GL}_{2} \mathbb{R} \rightarrow \mathrm{GL}(V)$ with

$$
\left(\begin{array}{ll}
a & b \\
c & d
\end{array}\right)^{\omega}=\left(\begin{array}{cccc}
a^{3} & a^{2} b & a b^{2} & b^{3} \\
3 a^{2} c & 2 a b c+a^{2} d & 2 a b d+b^{2} c & 3 b^{2} d \\
3 a c^{2} & 2 a c d+b c^{2} & 2 b c d+a d^{2} & 3 b d^{2} \\
c^{3} & c^{2} d & c d^{2} & d^{3}
\end{array}\right)
$$

equals the full group of automorphisms of $\mathscr{S}$, see [21], 73.19(b).

(b) We define a one-parameter group of linear maps by

$$
\varphi(r)(x, y, u, v)^{t}=(r x, r y,(r-1) x+u,(r-1) y+v)^{t},
$$

where $r \in \mathbb{R}, r>0$. The action of $\varphi(r)$ on $\mathscr{S} \backslash\{S\}$ is given by

$$
\left|\begin{array}{ll}
a & b \\
c & d
\end{array}\right|^{\varphi(r)}=\left|\begin{array}{cc}
1+(a-1) / r & b / r \\
c / r & 1+(d-1) / r
\end{array}\right| .
$$

Thus, $\varphi(r)$ leaves invariant the subspace

$$
E=\left|\begin{array}{ll}
1 & 0 \\
0 & 1
\end{array}\right| \in \mathscr{S}_{2}
$$


Moreover, $\varphi(r)$ fixes $S$ pointwise. We denote by $\mathscr{S}^{(r)}$ the image of $\mathscr{S}$ under the $\operatorname{map} \varphi(r)$.

(c) Let $\left(r_{i}\right)_{i}$ be a monotone decreasing sequence with $r_{i} \in \mathbb{R}_{>0}$ and $\lim _{i \rightarrow \infty} r_{i}=0$ and let $\left(L^{\left(r_{i}\right)}\right)_{i}$ with $L^{\left(r_{i}\right)} \in \mathscr{S}^{\left(r_{i}\right)}$ be a sequence which converges in $\mathrm{G}_{l}(V)$ to some 2-dimensional subspace $K$ of $V$. We will prove in part (d) that $K$ is an element of the classical complex spread

$$
\mathscr{S}_{\mathbb{C}}:\{S\} \cup\left\{\left|\begin{array}{cc}
a & -b \\
b & a
\end{array}\right| \mid a, b \in \mathbb{R}\right\}
$$

on $V$, and, conversely, that every element of $\mathscr{S}_{\mathbb{C}}$ is the limit of such a sequence.

This implies that $\mathscr{S}_{\mathbb{C}}$ is the Hausdorff limit $\mathrm{H}-\lim _{r \rightarrow 0} \mathscr{S}^{(r)}$ of the family $\left\{\mathscr{S}^{(r)}\right\}_{r>0}$. In particular, this shows assertion (2) of the theorem.

(d) We retain the notation of (c) and set $L_{i}:=\varphi\left(r_{i}\right)^{-1}\left(L^{\left(r_{i}\right)}\right)$. Since $L_{i}$ is an element of the compact spread $\mathscr{S}$ for every $i$, the sequence $\left(L_{i}\right)_{i}$ has an accumulation point $L \in \mathscr{S}$ and by passing to an appropriate subsequence we may assume that $\lim _{i \rightarrow \infty} L_{i}=L$ in $\mathrm{G}_{2}\left(\mathbb{R}^{4}\right)$. Consider the case $L \neq E$ first. Then $L \cap E=\{0\}$ (since $L, E \in \mathscr{S}$ ) and thus $L_{i} \cap E=\{0\}$ holds for almost all $i$, because $\lim _{i \rightarrow \infty} L_{i}=L$. For sufficiently large $i$ we thus may express $L_{i}$ in its Grassmann coordinates $A_{i}$ with respect to the decomposition $V=S \times E$. (This means that $L_{i}=\left\{\left(u, A_{i} u\right) \mid u \in S\right\} \leqslant S \times E$; the matrices $A_{i}$ are considered as linear maps $A_{i}: S \rightarrow E$, cf. [21, 64.3].) Observe that the Grassman coordinates $A^{\left(r_{i}\right)}$ of $L^{\left(r_{i}\right)}$ satisfy $A^{\left(r_{i}\right)}=r_{i} \cdot A_{i}$. Moreover, the entries of $A_{i}$ are bounded, because $\lim _{i \rightarrow \infty} A_{i}=A$, where $A$ is the matrix of Grassmann coordinates of $L$. This implies $\lim _{i \rightarrow \infty} A^{\left(r_{i}\right)}=0$ (since $\lim _{i \rightarrow \infty} r_{i}=0$ ) and thus $\lim _{i \rightarrow \infty} L^{\left(r_{i}\right)}=S$. Hence, $K=S$ indeed in an element of $\mathscr{S}_{\mathbb{C}}$.

Therefore, we may assume $\lim _{i \rightarrow \infty} L_{i}=E$. This implies that $L_{i}$ is contained in the open subset $\mathscr{S}_{2}$ for $\mathscr{S}$ for almost all $i$. For this reason there exist sequences $\left(b_{i}\right)_{i}$ and $\left(d_{i}\right)_{i}$ of real numbers with $d_{i}>0$ such that

$$
L_{i}=\left|\begin{array}{cc}
-3 b_{i}^{2}+d_{i}^{2} & 2 b_{i} \\
-2 b_{i}^{3}-2 b_{i} d_{i}^{2} & b_{i}^{2}+d_{i}^{2}
\end{array}\right| .
$$

From $\lim _{i \rightarrow \infty} L_{i}=E$ we derive $\lim _{i \rightarrow \infty} b_{i}=0$ and $\lim _{i \rightarrow \infty} d_{i}=1$. Moreover, we get

$$
\begin{aligned}
L^{\left(r_{i}\right)}=\varphi\left(r_{i}\right)\left(L_{i}\right) & =\left|\begin{array}{cc}
1+\frac{d_{i}^{2}-1}{r_{i}}-3 \frac{b_{i}}{r_{i}} b_{i} & 2 \frac{b_{i}}{r_{i}} \\
-2 \frac{b_{i}}{r_{i}}\left(b_{i}^{2}+d_{i}^{2}\right) & 1+\frac{d_{i}^{2}-1}{r_{i}}+\frac{b_{i}}{r_{i}} b_{i}
\end{array}\right| \\
& =\left|\begin{array}{cc}
1+y_{i}-3 x_{i} b_{i} & 2 x_{i} \\
-2 x_{i}\left(b_{i}^{2}+d_{i}^{2}\right) & 1+y_{i}+x_{i} b_{i}
\end{array}\right|,
\end{aligned}
$$

where $x_{i}=b_{i} / r_{i}$ and $y_{i}=\left(d_{i}^{2}-1\right) / r_{i}$. Notice that the limit $K$ of $\left(L^{\left(r_{i}\right)}\right)_{i}$ is skew to $S$ if and only if the Grassmann coordinates of $L^{\left(r_{i}\right)}$ (with respect to $W$ and $S$ ) converge. In this case, both limits $\xi:=\lim _{i \rightarrow \infty} x_{i}$ and $\eta:=\lim _{i \rightarrow \infty} y_{i}$ exist. This implies that

$$
K=\lim _{i \rightarrow \infty} L^{\left(r_{i}\right)}=\left|\begin{array}{cc}
1+\eta & 2 \xi \\
-2 \xi & 1+\eta
\end{array}\right| \in \mathscr{S}_{\mathbb{C}}
$$


Indeed, these arguments also show that every classical line $L \in \mathscr{S}_{\mathbb{C}}$ can be written as $\lim _{i \rightarrow 0} L^{(r)}$ with $L^{(r)} \in \mathscr{S}^{(r)}$.

It remains to consider the case that $K$ intersects nontrivially with $S$. Then at least one of the sequence $\left(\left|x_{i}\right|\right)_{i}$ and $\left(\left|y_{i}\right|\right)_{i}$ is not bounded. Assume first that there are infinitely many indices $i$ with $\left|x_{i}\right| \leqslant\left|y_{i}\right|$. By passing to subsequences again, we may assume the following situation:

(a) $\left|x_{i}\right| \leqslant\left|y_{i}\right|$ holds for every $i$.

(b) $\lim _{i \rightarrow \infty}\left|y_{i}\right|=\infty$, and

(c) $\lim _{i \rightarrow \infty} x_{i} / y_{i}=\alpha \in \mathbb{R}$.

Then we may write

$$
L^{\left(r_{i}\right)}=\mathbb{R} \cdot\left(\begin{array}{c}
y_{i}^{-1} \\
0 \\
\frac{1}{y_{i}}+1-3 \frac{x_{i}}{y_{i}} b_{i} \\
-2 \frac{x_{i}}{y_{i}}\left(b_{i}^{2}+d_{i}^{2}\right)
\end{array}\right) \oplus \mathbb{R}\left(\begin{array}{c}
0 \\
y_{i}^{-1} \\
2 \frac{x_{i}}{y_{i}} \\
\frac{1}{y_{i}}+1+\frac{x_{i}}{y_{i}} b_{i}
\end{array}\right)
$$

and end up with

$$
K=\lim _{i \rightarrow \infty} L^{\left(r_{i}\right)}=\mathbb{R} \cdot\left(\begin{array}{c}
0 \\
0 \\
1 \\
-2 \alpha
\end{array}\right) \oplus \mathbb{R} \cdot\left(\begin{array}{c}
0 \\
0 \\
2 \alpha \\
1
\end{array}\right)=S .
$$

The same arguments show that $\lim _{i \rightarrow \infty} L^{\left(r_{i}\right)}=S$ if $\left|x_{i}\right| \geqslant\left|y_{i}\right|$ holds for almost all $i$. Summing up we have shown that $\lim _{i \rightarrow \infty} L^{\left(r_{i}\right)}$ is a classical line. This finishes the proof of the assertion stated in (c).

(e) It remains to show part (3), i.e. we have to determine the limit group $\Gamma^{*}$ of the automorphism groups $\Gamma^{(r)}=\varphi(r)\left\lceil\varphi(r)^{-1}\right.$ of the spreads $\mathscr{S}^{(r)}$. Obviously, it suffices to compute the limit group $\Delta^{*}$ of the groups $\mathrm{S} \Gamma^{(r)}=\left\{\gamma \in\left\lceil^{(r)} \mid \operatorname{det} \gamma=1\right\}\right.$, since $\Gamma^{*}$ is generated by $\Delta^{*}=\mathrm{S} \Gamma^{*}=\left\{\gamma \in \Gamma^{*} \mid \operatorname{det} \gamma=1\right\}$ together with the real multiples of the unit matrix. We claim that

$$
\Delta^{*}=\left\{\left(\begin{array}{cccc}
\cos t & -\sin t & & \\
\sin t & \cos t & & \\
x & -y & \cos 3 t & -\sin 3 t \\
y & x & \sin 3 t & \cos 3 t
\end{array}\right) \mid t, x, y \in \mathbb{R}\right\} \cong \mathrm{SO}_{2} \mathbb{R} \ltimes \mathbb{R}^{2} .
$$

The Lie algebra of $\mathrm{S}\left\lceil\right.$ is the subalgebra $\mathfrak{g}=\mathrm{T}_{e}(\mathrm{~S}\lceil)=\mathbb{R} \cdot X \oplus \mathbb{R} \cdot Y \oplus \mathbb{R} \cdot Z$ of $\mathfrak{s l}_{4} \mathbb{R}$, where

$$
X=\left(\begin{array}{cccc}
0 & 1 & & \\
-3 & 0 & 2 & \\
& -2 & 0 & 3 \\
& & -1 & 0
\end{array}\right), Y=\left(\begin{array}{llll}
0 & 1 & & \\
& 0 & 2 & \\
& & 0 & 3 \\
& & & 0
\end{array}\right), Z=\left(\begin{array}{cccc}
3 & & & \\
& 1 & & \\
& & -1 & \\
& & & -3
\end{array}\right) \text {. }
$$


In order to prove the assertion, we show that the Hausdorff limit $\mathrm{H}$ $\lim _{r \rightarrow 0} \varphi(r) \mathfrak{g} \varphi(r)^{-1}$ is just

$$
\mathfrak{h}:=\left\{\left(\begin{array}{cccc}
0 & a & & \\
-a & 0 & & \\
b & c & 0 & -3 a \\
-c & b & -3 a & 0
\end{array}\right) \mid a, b, c \in \mathbb{R}\right\} .
$$

Computing the limits of the one-dimensional subalgebras

$$
\begin{aligned}
& \mathfrak{r}^{(r)}=\mathbb{R} \cdot \varphi(r) X \varphi(r)^{-1}= \mathbb{R} \cdot\left(\begin{array}{cccc}
0 & 1 & 0 & 0 \\
-1-2 r & 0 & 2 r & 0 \\
0 & -2 & 0 & 3 \\
2-2 r & 0 & -3+2 r & 0
\end{array}\right) \\
& \mathfrak{n}^{(r)}=\mathbb{R} \cdot \varphi(r) Y \varphi(r)^{-1}= \mathbb{R} \cdot\left(\begin{array}{cccc}
0 & r & 0 & 0 \\
-2 r^{2}+2 r & 0 & 2 r^{2} & 0 \\
0 & 2-2 r & 0 & 3 r \\
-2(r-1)^{2} & 0 & 2 r^{2}-2 r & 0
\end{array}\right) \\
& \mathfrak{j}^{(r)}=\mathbb{R} \cdot \varphi(r) Z \varphi(r)^{-1}=\mathbb{R} \cdot\left(\begin{array}{cccc}
3 r & 0 & \\
0 & r & & \\
4 r-4 & 0 & -r & 0 \\
0 & 4 r-4 & 0 & -3 r
\end{array}\right)
\end{aligned}
$$

we eventually obtain

$$
\begin{aligned}
\mathrm{H}-\lim _{r \rightarrow 0} \mathrm{r}^{(r)} & =\mathbb{R} \cdot\left(\begin{array}{cccc}
0 & 1 & & \\
-1 & 0 & & \\
0 & -2 & 0 & 3 \\
2 & 0 & -3 & 0
\end{array}\right), \\
\mathrm{H}-\lim _{r \rightarrow 0} \mathfrak{r}^{(r)} & =\mathbb{R} \cdot\left(\begin{array}{cccc}
0 & 0 & & \\
0 & 0 & & \\
0 & 2 & 0 & 0 \\
-2 & 0 & 0 & 0
\end{array}\right), \\
\mathrm{H}-\lim _{r \rightarrow 0} \mathfrak{z}^{(r)} & =\mathbb{R} \cdot\left(\begin{array}{cccc}
0 & 0 & & \\
0 & 0 & & \\
-4 & 0 & 0 & 0 \\
0 & -4 & 0 & 0
\end{array}\right) .
\end{aligned}
$$

Consequently, every accumulation point of the three-dimensional subspaces $\mathfrak{g}^{\varphi(r)}$ equals $\mathfrak{h}$. This completes the proof of the theorem. 


\section{Proof of Theorem B}

In this section, let $\mathscr{S}=(P, \mathscr{L}, \mathscr{F})$ be a $2 l$-dimensional smooth stable plane with tangent bundle $\left(\mathrm{T} P, P, \tau_{P}\right)$ and associated Grassman bundle $(\mathrm{G} P, P, g)$. For $p \in P$, let $\mathrm{K}_{p} P=\mathrm{K}_{l}\left(\mathrm{~T}_{p} P\right)$ be the set of compact subsets of the Grassmannian $\mathrm{G}_{l}\left(\mathrm{~T}_{p} P\right)$ and let $\left.\mathrm{S}_{p} P=\mathrm{S}_{l} \mathrm{~T}_{p} P\right)$ be the set of compact spreads on $\mathrm{T}_{p} P$.

We are going to define topological locally trivial bundles $(\mathrm{K} P, P, k)$ and $(\mathrm{S} P, P, s)$. Set $\mathrm{K} P=\cup_{p \in P} \mathrm{~K}_{p} P$ and let $k: \mathrm{K} P \rightarrow P$ be the canonical projection. We topologize $\mathrm{K} P$ by constructing charts $k^{-1}(U) \rightarrow U \times \mathrm{K}_{l}\left(\mathbb{R}^{2 l}\right)$ for any chart $\left(f_{G}, U\right): g^{-1}(U) \rightarrow U \times \mathrm{G}_{l}\left(\mathbb{R}^{2 l}\right): x \mapsto\left(f_{G}^{1}(x), f_{G}^{2}(x)\right)=\left(g(x), f_{G}^{2}(x)\right)$ of the Grassmann bundle $(\mathrm{G} P, P, g)$ : we take $\left(f_{K}, U\right): k^{-1}(U) \rightarrow U \times \mathrm{K}_{l}\left(\mathbb{R}^{2 l}\right): X \mapsto\left(f_{K}^{1}(X)\right.$, $\left.f_{K}^{2}(X)\right):=\left(k(X), f_{G}^{2}(X)\right)$ as a bundle chart for $(\mathrm{K} P, P, k)$. Clearly, for charts $\left(f_{K}, U\right)$ and $\left(f_{K}^{\prime}, U^{\prime}\right)$ the transition map $f_{K} \circ f_{K}^{\prime-1}$ is continuous. Thus, the family

$$
\left\{\left(f_{K}, U\right) \mid\left(f_{G}, U\right) \text { is a bundle chart of }(\mathrm{G} P, P, g)\right\}
$$

defines a bundle atlas for $(\mathrm{K} P, P, k)$, which turns $(\mathrm{K} P, P, k)$ into a locally trivial topological bundle with fibres homeomorphic to $\mathrm{K}_{l}\left(\mathbb{R}^{2 l}\right)$. Finally, we may consider $(\mathrm{S} P, P, s)$ as a subbundle of $(\mathrm{K} P, P, k)$, since the transition maps are induced by linear mappings. From the description of convergence with respect to the Hausdorff topology, see section 2, we immediately get the following lemma.

4.1 Lemma. A sequence $\left(X_{n}\right)_{n \in \mathbb{N}}$ in $\mathrm{K} P$ converges to $X \in \mathrm{K} P$ if and only if the sequence $\left(k\left(X_{n}\right)\right)_{n}$ converges to $k(X)$ in $P$ and $\mathrm{H}-\lim _{i \rightarrow \infty} f_{G}^{2}\left(X_{n}\right)=f_{G}^{2}(X)$ for some chart $\left(f_{G}, U\right)$ of $(\mathrm{K} P, P, k)$ with $k(X) \in U$.

We proceed with the following lemma, which is the key to the proof of Theorem B. We denote by $(\mathrm{V} P, P, v)$ the frame bundle over $P$, see [10, §7].

4.2 Lemma. The map $\omega_{1}: \mathscr{F} \rightarrow \mathrm{GP}:(p, L) \mapsto \mathrm{T}_{p} L$ is smooth.

Proof. Let $(p, L)$ be some flag. The projections $\pi_{P}: \mathscr{F} \rightarrow P$ and $\pi_{\mathscr{L}}: \mathscr{F} \rightarrow \mathscr{L}$ are submersions by [3, p. 308]. Thus the kernel $\mathscr{K}:=\operatorname{kerD} \pi_{\mathscr{L}}$ is a locally trivial subbundle of the tangent bundle T $\mathscr{F}$, see Husemoller, [10, Th. 8.2], and there is an open neighborhood $U$ of $(p, L)$ in $\mathscr{F}$ such that the restriction of $\mathscr{K}$ to $U$ is a trivial bundle. Hence we can choose a family $\mathscr{X}=\left(X_{i}\right)_{i=1}^{l}$ of linearly independent vector fields on $U$ having values in $\mathscr{K}$. Let $\pi_{2 l, l}^{r}: V_{l}\left(\mathrm{~T}_{r} P\right) \rightarrow \mathrm{G}_{l}\left(\mathrm{~T}_{r} P\right):\left(v_{1}, \ldots, v_{l}\right) \mapsto$ $\left\langle v_{1} \ldots, v_{l}\right\rangle$ be the (smooth) projection from the Stiefel manifold $\mathrm{V}_{l}\left(\mathrm{~T}_{r} P\right)$ of $l$-frames in $\mathrm{T}_{r} P$ onto the Grassmannian $\mathrm{G}_{l}\left(\mathrm{~T}_{r} P\right)$. The projection $\pi_{2 l, l}^{r}$ induces in the natural way a smooth bundle projection $\pi_{2 l, l}: \mathrm{V} P \rightarrow \mathrm{G} P$, where $(\mathrm{V} P, P, v)$ denotes the frame bundle over $P$ and $(\mathrm{G} P, P, g)$ is the Grassmann bundle over $P$. By [3], (3.2), the composition

$$
\omega_{U}: U \stackrel{\mathscr{x}}{\longrightarrow} \prod_{i=1}^{l} \mathscr{K} \stackrel{\prod_{i=1}^{l} \mathrm{D} \pi_{P}}{\longrightarrow} \mathrm{V} P \stackrel{\pi_{2 l, l}}{\longrightarrow} \mathrm{GP}
$$

is a smooth map which maps each flag $(r, K) \in U$ to the tangent subspace $\mathrm{T}_{r} K \in \mathrm{G}_{l}\left(\mathrm{~T}_{r} P\right)$. In particular, we have $\left.\omega_{1}\right|_{U}=\omega_{U}$, i.e. the map $\omega_{1}$ is smooth around $(p, L)$. This proves the lemma. 
The map $\omega_{1}$ induces another map

$$
\omega_{2}: P \rightarrow \mathrm{K} P: r \mapsto \omega_{1}\left(r, \mathscr{L}_{r}\right)=\left\{\mathrm{T}_{r} K \mid K \in \mathscr{L}_{r}\right\}=\mathscr{S}_{r} .
$$

We claim that $\omega_{2}$ is continuous on $P$. To see this, choose $r \in P$ and a compact neighborhood $U$ of $r$. Let $\left(r_{i}\right)_{i \in \mathbb{N}}$ be some sequence in $U$ converging to $r$. By Lemma (4.1) we have to show that the sequence $\left(\mathscr{S}_{r_{i}}\right)_{i}$ converges to $\mathscr{S}_{r}$ in $\mathrm{K} P$. So take $\mathrm{T}_{r} K \in \mathscr{S}_{r}$ and choose a sequence $\left(K_{i}\right)_{i}$ of lines through $r_{i}$ with $\lim _{i \rightarrow \infty} K_{i}=K$. Since $\omega_{1}$ is continuous, we get

$$
\lim _{i \rightarrow \infty} \mathrm{T}_{r_{i}} K_{i}=\lim _{i \rightarrow \infty} \omega_{1}\left(r_{i}, K_{i}\right)=\omega_{1}(r, K)=\mathrm{T}_{r} K .
$$

On the other hand, suppose that there is a sequence $\left(\mathrm{T}_{r_{i}} K_{i}\right)_{i} \in \mathscr{S}_{r_{i}}$, which has a cluster point outside $\mathscr{S}_{r}$. By passing to some subsequence we may assume that $\lim _{i \rightarrow \infty}\left(\mathrm{T}_{r_{i}} K_{i}\right)_{i}$ exists and lies outside $\mathscr{S}_{r}$. Since $U$ and all line pencils are compact and the space $\mathscr{F}$ of flags is closed in the product $P \times \mathscr{L}$ (see Löwen, [15, (1.17)]), the set $\mathscr{F} \cap(U \times \mathscr{L})$ is compact as well. Thus we find a subsequence $\left(K_{i}^{\prime}\right)_{i}$ of $\left(K_{i}\right)_{i}$, which converges to some line $K$. This gives $\lim _{i \rightarrow \infty}\left(r_{i}^{\prime}, K_{i}^{\prime}\right)=(r, K) \in \mathscr{F}$ and continuity of $\omega_{1}$ yields the contradiction

$$
\lim _{i \rightarrow \infty} \mathrm{T}_{r_{i}^{\prime}} K_{i}^{\prime}=\lim _{i \rightarrow \infty} \omega_{1}\left(r_{i}^{\prime}, K_{i}^{\prime}\right)=\omega_{1}(r, K)=\mathrm{T}_{r} K \in \mathscr{S}_{r},
$$

which shows that $\left(\mathscr{S}_{r_{i}}\right)_{i}$ converges to $\mathscr{S}_{r}$. By definition, the image of $\omega_{2}$ is in fact contained in $\mathrm{S} P$. Hence, we have proved

4.3 Theorem. The map $\omega_{2}: P \rightarrow S P: r \mapsto \omega_{1}\left(r, \mathscr{L}_{r}\right)=\mathscr{S}_{r}$ is a continuous section of the bundle $(S P, P, s)$.

At this stage it would be natural to define a moduli bundle $\mathrm{J} P$ as a quotient of $\mathrm{S} P$ and pass from the section $\omega_{2}$ to some section $\omega_{3}$ of $\mathrm{J} P$ in order to prove Theorem B. However, this step is not necessary, as the following construction shows.

Let $V$ be a real vector space of dimension $2 l$. For any point $p \in P$ we choose a linear isomorphism from $\mathrm{T}_{p} P$ to $V$. These isomorphisms induce homeomorphisms $\vartheta_{P}$ from $\mathrm{G}_{l}\left(\mathrm{~T}_{p} P\right)$ to $\mathrm{G}_{l}(V)$. Now, consider the map $\omega: P \rightarrow \mathrm{J}_{l}(V)$ : $p \mapsto\left[\vartheta_{p}\left(\omega_{2}(p)\right)\right]$, where square brackets denote the isomorphism type of a spread. Since the isomorphism classes of spreads on $V$ are invariant under linear isomorphisms of $V$, the map $\omega$ is independent of the choice of the maps $\vartheta_{p}, p \in P$. Take a local trivialization $\left(f_{S}, U\right): s^{-1}(U) \rightarrow U \times S_{l}(V): X \mapsto\left(f_{S}^{1}(X), f_{S}^{2}(X)\right)$ of $(\mathrm{S} P, P, s)$, where $f_{S}^{2}$ is induced by linear isomorphisms between the tangent spaces $\mathrm{T}_{r} P$ and $V$. Hence, we have $\omega(r)=\left[f_{S}^{2}\left(\omega_{2}(r)\right)\right]=\left[f_{S}^{2}\left(\mathscr{S}_{r}\right)\right]$, which shows that $\omega$ is continuous on $U$ and thus on $P$. In particular, the continuity of the map $\omega: P \rightarrow \mathrm{J}_{l}(V)$ exactly defines and proves the assertion of Theorem B.

\section{Proof of Theorem C}

In order to construct examples of smooth affine planes $\mathscr{A}$ with non-isomorphic tangent translation planes at two points, we take a division algebra $\mathbb{F} \in\{\mathbb{C}, \mathbb{H}, \mathbb{O}\}$. Put $l:=\operatorname{dim}_{\mathbb{R}} \mathbb{F}$. Let $A=\mathbb{F}^{2}$ be the set of points of $\mathscr{A}$. The lines of $\mathscr{A}$ (regarded as 
subsets of $A$ ) are described by

$$
\begin{aligned}
L_{c} & :=\left\{\left(x_{1}, x_{2}\right) \in A \mid x_{1}=c\right\}, \\
L_{a . b} & :=\left\{\left(x_{1}, x_{2}\right) \in A \mid x_{2}=a x_{1}+b+\operatorname{th}\left(x_{1}, a\right)\right\},
\end{aligned}
$$

where $t \in \mathbb{R}$ and $a, b, c \in \mathbb{F}$, and $h: \mathbb{F}^{2} \rightarrow \mathbb{F}$ denotes some fixed smooth function with compact support. This gives an incidence structure $\mathscr{A}:=\mathscr{A}(h, t)=(A, \mathscr{K})$. For small values of $|t|$ we get in fact an affine plane:

5.1 Lemma. There is a neighborhood I of 0 in $\mathbb{R}$ such that $\mathscr{A}=\mathscr{A}(h, t)$ is an affine plane for any $t \in I$.

Proof. We start by showing that any two distinct points $\left(x_{1}, x_{2}\right),\left(y_{1}, y_{2}\right) \in \mathbb{F}^{2}$ lie on a unique line. This is obviously true if $x_{1}=y_{1}$. So let $x_{1} \neq y_{1}$. We have to verify that there are uniquely determined numbers $a, b \in \mathbb{F}$ satisfying

$$
\begin{aligned}
& x_{2}=a x_{1}+b+\operatorname{th}\left(x_{1}, a\right), \\
& y_{2}=a y_{1}+b+\operatorname{th}\left(y_{1}, a\right) .
\end{aligned}
$$

This is eqivalent to showing that $x_{2}-y_{2}=a\left(x_{1}-y_{1}\right)+t\left(h\left(x_{1}, a\right)-h\left(y_{1}, a\right)\right)$ has a unique solution $a \in \mathbb{F}$ and it suffices to prove that the maps

$$
p_{x_{1}, y_{1}}: \mathbb{F} \rightarrow \mathbb{F}: a \mapsto a\left(x_{1}-y_{1}\right)+t\left(h\left(x_{1}, a\right)-h\left(y_{1}, a\right)\right)
$$

are bijective for any $x_{1}, y_{1} \in \mathbb{F}$ with $x_{1} \neq y_{1}$, if $|t|$ is sufficiently small. The derivative of $p_{x_{1}, y_{1}}$ in $a$ is given by

$$
\mathrm{D} p_{x_{1}, y_{1}}(a)=\left|x_{1}-y_{1}\right|\left(C_{x_{1}, y_{1}}+\frac{t}{\left|x_{1}-y_{1}\right|}\left(\mathrm{D}_{2} h\left(x_{1}, a\right)-\mathrm{D}_{2} h\left(y_{1}, a\right)\right)\right),
$$

where $C_{x_{1}, y_{1}}$ is the (real) matrix that describes the (orthogonal) $\mathbb{R}$-linear map $\mathbb{F} \rightarrow \mathbb{F}: a \mapsto a\left(x_{1}-y_{1}\right)\left|x_{1}-y_{1}\right|^{-1}$. With $m:=\sup _{(x, d) \in \mathbb{F}^{2}}\left\|\mathrm{D}\left(\mathrm{D}_{2} h(x, d)\right)\right\|$ we get

$$
\left\|\frac{t}{\left|x_{1}-y_{1}\right|}\left(\mathrm{D}_{2} h\left(x_{1}, a\right)-\mathrm{D}_{2} h\left(y_{1}, a\right)\right)\right\| \leqslant \frac{|t|}{\left|x_{1}-y_{1}\right|} m \cdot\left\|\left(x_{1}, a\right)-\left(y_{1}, a\right)\right\|=|t| \cdot m \text {. }
$$

Let $B \subseteq$ Mat $_{l} \mathbb{R}$ denote the closed ball around the origin with radius $m$. Since the continuous map

$$
g:\left(O_{l} \mathbb{R} \times B\right) \times \mathbb{R} \rightarrow \mathrm{Mat}_{l} \mathbb{R}:((O, M), t) \mapsto O+t \cdot M
$$

maps $\left(O_{l} \mathbb{R} \times B\right) \times\{0\}$ on $O_{l} \mathbb{R} \subseteq \mathrm{GL}_{l} \mathbb{R}$, the compactness of $O_{l} \mathbb{R} \times B$ implies that there is an interval $I \subseteq \mathbb{R}$ centered at 0 such that $g\left(\left(O_{l} \mathbb{R}\right) \times I\right) \subseteq \mathrm{GL}_{l} \mathbb{R}$. Note that the interval $I$ does not depend on the choices of $x_{1}, y_{1} \in \mathbb{F}$ and of $a \in \mathbb{F}$. For the rest of the proof let $t \in I$. Then $\mathrm{D}_{x_{1}, y_{1}}(a) \in \mathrm{GL}_{l} \mathbb{R}$ for any $a \in \mathbb{F}$. Since the function $h$ has compact support, the map $a \mapsto \mathrm{D} p_{x_{1}, y_{1}}(a)$ is constant outside a compactum. Thus, according to the Hadamard criterion (see [21, 74.19]), the map $p_{x_{1}, y_{1}}$ is a diffeomorphism for any distinct $x_{1}, y_{1} \in \mathbb{F}$. This shows that any two distinct points are joined by a unique line (if $t \in I$ ). Next, we verify that any two non-parallel lines meet in exactly one point. Disregarding the trivial cases, this is equivalent to showing that for $t \in I$ the system

$$
\begin{aligned}
& x_{2}=a x_{1}+b+t \cdot h\left(x_{1}, a\right) \\
& x_{2}=c x_{1}+d+t \cdot h\left(x_{1}, c\right),
\end{aligned}
$$


where $a, b, c, d \in \mathbb{F}$ with $a \neq c$, has a unique solution $\left(x_{1}, x_{2}\right) \in \mathbb{F}^{2}$. As before it suffices to check whether the map

$$
q_{a, c}: \mathbb{F} \rightarrow \mathbb{F}: x_{1} \mapsto(a-c) x_{1}+t\left(h\left(x_{1}, a\right)-h\left(x_{1}, c\right)\right)
$$

is bijective. Since the verification of this fact runs along the same lines as before, we omit a proof. Due to what we have proved so far, it is also clear that $\mathscr{A}$ satisfies the parallel axiom. Hence, we have shown that $\mathscr{A}$ is an affine plane.

Remark. In [18], Otte constructs non-classical smooth affine translation planes by disturbing the multiplication of the classical division algebras by some smooth map with compact support.

We are going to show that the affine planes $\mathscr{A}(h, t)$ just defined are even smooth affine planes, i.e. joining, interesecting, and drawing parallels are smooth maps. For the set $A=\mathbb{F}^{2}$ of points we take the ordinary real smooth structure and we endow the set $\mathscr{K}$ of lines with a smooth structure simply by identifying each line of $\mathscr{A}$ with the "classical" line (of $\mathscr{A}_{2} \mathbb{F}$ ) with which it coincides outside a compact set. In the proof of the following theorem we will omit the verification of the smoothness of drawing parallels, since we are mainly interested in the underlying structure of a smooth stable plane.

5.2 Proposition. The affine planes $\mathscr{A}(t, h)$ are smooth affine planes.

Proof. 1) We show that the join map is smooth. Let $\left(\tilde{x}_{1}, \tilde{x}_{2}\right),\left(\tilde{y}_{1}, \tilde{y}_{2}\right) \in \mathbb{F}^{2}$. We start by considering the case $\tilde{x}_{1} \neq \tilde{y}_{1}$. Choose two disjoint neighborhoods $U$ and $V$ of $\left(\tilde{x}_{1}, \tilde{x}_{2}\right)$ and $\left(\tilde{y}_{1}, \tilde{y}_{2}\right) \in \mathbb{F}^{2}$, respectively, such that $x_{1} \neq y_{1}$ for any $\left(x_{1}, x_{2}\right) \in U$ and $\left(y_{1}, y_{2}\right) \in V$. This guarantees that the line $L=L\left(x_{1}, x_{2}, y_{1}, y_{2}\right)$ joining the point $\left(x_{1}, x_{2}\right)$ with $\left(y_{1}, y_{2}\right)$ is not a vertical line. Hence, the line $L$ is determined by the following equations

$$
\begin{aligned}
& x_{2}=a x_{1}+b+t \cdot h\left(x_{1}, a\right) \\
& y_{2}=a y_{1}+b+t \cdot h\left(x_{1}, a\right) .
\end{aligned}
$$

We have to show that the coefficients $a, b \in \mathbb{F}$ depend smoothly on the points $\left(x_{1}, x_{2}\right)$ and $\left(y_{1}, y_{2}\right)$. It is sufficient to prove that the parameter $a \in \mathbb{F}$ defined by

$$
x_{2}-y_{2}=a\left(x_{1}-y_{1}\right)+t\left(h\left(x_{1}, a\right)-h\left(y_{1}, a\right)\right)
$$

is a smooth function in $\left(x_{1}, x_{2}, y_{1}, y_{2}\right)$ on $U \times V$. In order to apply the implicit function theorem we consider the smooth map

$$
F: \mathbb{F}^{5} \rightarrow \mathbb{F}:\left(x_{1}, x_{2}, y_{1}, y_{2}, a\right) \mapsto y_{2}-x_{2}+a\left(x_{1}-y_{1}\right)+t\left(h\left(x_{1}, a\right)-h\left(y_{1}, a\right)\right) .
$$

The partial derivative $\mathrm{D}_{5} F$ is given by

$$
\mathrm{D}_{5} F\left(x_{1}, x_{2}, y_{1}, y_{2}, a\right)=\left|x_{1}-y_{1}\right|\left(C_{x_{1}, y_{1}}-\frac{t}{\left|x_{1}-y_{1}\right|}\left(\mathrm{D}_{2} h\left(x_{1}, a\right)-\mathrm{D}_{2} h\left(y_{1}, a\right)\right)\right),
$$

which is regular for $t \in I$ according to the proof of the preceding lemma. Thus, the values $a$ and $b$ depend smoothly on $\left(x_{1}, x_{2}, y_{1}, y_{2}\right)$. 
Now we turn to the case where $\tilde{x}_{1}=\tilde{y}_{1}$. Then we have $\tilde{x}_{2} \neq \tilde{y}_{2}$. We choose the disjoint neighborhoods $U$ and $V$ in such a way that every line joining a point of $U$ with a point of $V$ is given by

$$
c z_{2}=z_{1}+b+ \begin{cases}c t h\left(z_{1}, c^{-1}\right) & \text { if } c \neq 0 \\ 0 & \text { if } c=0 .\end{cases}
$$

The parameters $b$ and $c$ of a line joining $\left(x_{1}, x_{2}\right) \in U$ and $\left(y_{1}, y_{2}\right) \in V$ are determined by the equations

$$
\begin{aligned}
& c x_{2}=x_{1}+b+ \begin{cases}c t h\left(x_{1}, c^{-1}\right) & \text { if } c \neq 0 \\
0 & \text { if } c=0\end{cases} \\
& c y_{2}=y_{1}+b+ \begin{cases}c t h\left(y_{1}, c^{-1}\right) & \text { if } c \neq 0 \\
0 & \text { if } c=0 .\end{cases}
\end{aligned}
$$

Since $c$ tends to zero as $x_{1}$ tends to $\tilde{x}_{1}$ and $y_{1}$ tends to $\tilde{y}_{1}$, we may make $U$ and $V$ so small such that $\left(x_{1}, c^{-1}\right)$ and $\left(y_{1}, c^{-1}\right)$ are not contained in the support of $h$ for any possible $\left(x_{1}, x_{2}\right) \in U$ and any $\left(y_{1}, y_{2}\right) \in V$. Hence, the equations above reduce to

$$
c x_{2}=x_{1}+b, c y_{2}=y_{1}+b \text {, }
$$

and, trivially, $b$ and $c$ depend smoothly on $x_{1}, x_{2}, y_{1}, y_{2}$. This shows the smoothness of the join map.

2) The smoothness of the intersection map at some pair $(K, L)$ of lines can be checked in much the same way as in the first part of this proof by using again the implicit function theorem. As before, we have to distinguish between two cases $(K, L)=\left(L_{a, b}, L_{c, d}\right)$ with $a \neq b$ and $(K, L)=\left(L_{a, b}, L_{c}\right)$. This finishes the proof.

Remark. It is quite natural to ask whether or not there exist smooth structures on the point space and on the line space of the projective closure $\mathscr{P}=(P, \mathscr{L})$ of $\mathscr{A}$ such that $\mathscr{P}$ becomes a smooth projective plane. It seems likely that the answer is negative. However, removing the point $(\infty)$ at infinity which corresponds to the vertical lines, we do get a smooth punctured projective plane $\mathscr{P}^{\prime}:$ as each line of $\mathscr{A}$ coincides outside a compactum with a line of $\mathscr{A}_{2} \mathbb{F}$, it is possible to describe $\mathscr{P}$ by introducing homogeneous coordinates if $\mathbb{F}=\mathbb{C}, \mathbb{H}$. In the case $\mathbb{F}=\mathbb{O}$ homogeneous coordinates do not work any more, but still one can use affine charts for describing $\mathscr{P}_{2} \mathbb{O}$. In this way one gets smooth structures on $P$ and on $\mathscr{L}=\mathscr{K} \cup\left\{L_{\infty}\right\}$ such that $\mathscr{P}^{\prime}$ becomes a smooth punctured projective plane. Obviously, the tangent translation planes at points $p \neq(\infty)$ on the line $L_{\infty}$ at infinity are isomorphic to the classical affine plane $\mathscr{A}_{2} \mathbb{F}$.

Now we want to study the tangent translation plane $\mathscr{A}_{\left(y_{1}, y_{2}\right)}$ at some point $\left(y_{1}, y_{2}\right) \in A$. Except for the vertical line, every point row through $\left(y_{1}, y_{2}\right)$ is given by some equation

$$
x_{2}=F_{a}\left(x_{1}\right):=a x_{1}+\operatorname{th}\left(x_{1}, a\right)+y_{2}-a y_{1}-\operatorname{th}\left(y_{1}, a\right), a \in \mathbb{F} .
$$

In order to describe the tangent spread $\mathscr{S}_{\left(y_{1}, y_{2}\right)}$, which defines $\mathscr{A}_{\left(y_{1}, y_{2}\right)}$, in terms of Grassmann coordinates, we have to determine $\mathbb{R}$-linear maps whose graphs are the non-vertical elements of $\mathscr{S}_{\left(y_{1}, y_{2}\right)}$. These mappings are the derivatives $\mathrm{D} F_{a}\left(y_{1}\right)$ for any $a \in \mathbb{F}$. Let $A(a)$ denote the real $(l \times l)$-matrix that corresponds to the $\mathbb{R}$-linear map $x \mapsto a x: \mathbb{F} \rightarrow \mathbb{F}$. Then

$$
\mathrm{D} F_{a}\left(y_{1}\right)=A(a)+t \mathrm{D}_{1} h\left(y_{1}, a\right) .
$$


5.3 Proposition. The affine planes $\mathscr{A}(t, h)$ have smooth tangent translation planes at any point.

Proof. We identify the tangent space $\mathrm{T}_{\left(y_{1}, y_{2}\right)} A$ with $\mathscr{F}^{2}$. According to Otte [18, 5.14], the translation plane $\mathscr{A}_{\left(y_{1}, y_{2}\right)}$ is a smooth affine plane if and only if the map $\sigma: \mathbb{F}^{2} \backslash\{(0,0)\} \rightarrow \mathrm{G}_{l}\left(\mathbb{F}^{2}\right)$, which assigns to any non-zero vector $v$ the unique spread element that contains $v$, is smooth. On a sufficiently small neighborhood $U$ of some point $\left(0, \tilde{x}_{2}\right) \in \mathbb{F}^{2}$ with $\tilde{x}_{2} \neq 0$ the parameter $a$ given by $x_{2}=\mathrm{D} F_{a}\left(y_{1}\right) x_{1}$ (for $\left(x_{1}, x_{2}\right) \in U$ with $x_{1} \neq 0$ ) is not contained in the (compact) support of the map $a \mapsto \mathrm{D}_{1} h\left(y_{1}, a\right): \mathbb{F} \rightarrow \mathbb{F}$, since $a \rightarrow \infty$ if $\left(x_{1}, x_{2}\right)$ tends to $\left(0, \tilde{x}_{2}\right)$. Hence, in this case the parameter $a$ is simply given by $x_{2}=A(a) x_{1}=a x_{1}$ as in the classical plane $\mathscr{A}_{2} \mathbb{E}$. Thus $\sigma$ is smooth on $U$. It remains to check that $\sigma$ is smooth on the set $O:=\left\{\left(x_{1}, x_{2}\right) \in \mathbb{F}^{2} \mid x_{1} \neq 0\right\}$. Since $a \mapsto \mathrm{D} F_{a}\left(y_{1}\right)=A(a)+t \mathrm{D}_{1} h\left(y_{1}, a\right): \mathbb{F} \rightarrow \mathbb{F}$ is smooth, it suffices to show that the parameter $a$ defined by $x_{2}=A(a) x_{1}+$ $t \mathrm{D}_{1} h\left(y_{1}, a\right) x_{1}$ depends smoothly on $\left(x_{1}, x_{2}\right) \in O$. This is shown quite similarly to the proof of the foregoing proposition by using again the implicit function theorem.

Moreover, if we choose $y_{1}$ in the preceding proof large enough such that $\left(y_{1}, a\right)$ lies outside the support of $h$ for any $a \in \mathbb{F}$, then the tangent translation plane $\mathscr{A}_{\left(y_{1}, y_{2}\right)}$ is obviously just $\mathscr{A}_{2} \mathbb{F}$. Thus, in order to verify Theorem $C$, it suffices to prove the following proposition.

5.4 Proposition. There exists a smooth map $h: \mathbb{F} \rightarrow \mathbb{F}$ with compact support such that the tangent translation plane $\mathscr{A}_{\left(y_{1}, y_{2}\right)}$ is non-classical at some point $\left(y_{1}, y_{2}\right)$ of the smooth affine plane $\mathscr{A}(t, h), t \neq 0$.

Proof. Let $h_{1}, h_{2}: \mathbb{F} \rightarrow \mathbb{F}$ be two real-valued smooth functions with compact support such that $h:(y, a) \mapsto h_{1}(y) \cdot h_{2}(a): \mathbb{F}^{2} \rightarrow \mathbb{F}$ is not identically zero. Clearly, the map $h$ has compact support. Assume that the tangent spread $\mathscr{S}_{\left(y_{1}, y_{2}\right)}$ of $\mathscr{A}(t, h)$ is isomorphic to the classical spread $\mathscr{S}_{\mathbb{F}}$ for any $\left(y_{1}, y_{2}\right) \in \mathbb{F}^{2}$. We will show that then even $\mathscr{S}_{\mathbb{F}}=\mathscr{S}_{\left(y_{1}, y_{2}\right)}$. Of course, it suffices to check that $\mathscr{S}_{\mathbb{F}}$ is contained in $\mathscr{S}_{\left(y_{1}, y_{2}\right)}$. Since $\mathrm{D}_{1} h\left(y_{1}, a\right)=0$ for sufficiently large $a \in \mathbb{F}$, there exists a non-empty open set $U \subseteq \mathrm{G}_{l}(\mathbb{F})^{2}$ such that $\mathscr{S}_{\left(y_{1}, y_{2}\right)} \cap U=\mathscr{S}_{\mathbb{F}} \cap U$. We choose distinct elements $X, Y \in \mathscr{S}_{\mathbb{F}} \cap U$ and introduce Grassmann coordinates with respect to $X$ and $Y$. The Grassmann coordinates of any $S \in \mathscr{S}_{\mathbb{F}}$ are given by a real $l \times l$-matrix $M_{S}$. By [21, 64.8(b)], the set $\left\{M_{S} \mid S \in \mathscr{S}_{\mathbb{F}}\right\}$ is an affine subspace of $\mathbb{R}^{l \times l}$, and it is even a linear subspace because of $M_{X}=0$. Hence $\lambda M_{S}$ represents an element of $\mathscr{S}_{\mathbb{E}}$ for any $\lambda \in \mathbb{R}$. Since $U$ is a neighborhood of $X$, the matrix $\lambda M_{S}$ corresponds to an element of $\mathscr{S}_{\mathbb{F}} \cap U \subseteq \mathscr{S}_{\left(y_{1}, y_{2}\right)}$ for $|\lambda|$ sufficiently small. For any such $\lambda \neq 0$, the matrix $M_{S}=\lambda^{-1}\left(\lambda M_{S}\right)$ represents an element of $\mathscr{S}_{\left(y_{1}, y_{2}\right)}$, because $\mathscr{S}_{\left(y_{1}, y_{2}\right)}$ is isomorphic to the classical spread and contains $X$ and $Y$. Thus we have $\mathscr{S}_{\mathbb{E}} \subseteq \mathscr{S}_{\left(y_{1}, y_{2}\right)}$ for any $\left(y_{1}, y_{2}\right) \in \mathbb{F}^{2}$. Identifying $\mathbb{F}$ with $\mathbb{R}^{l}$ in the natural way, we have $\mathrm{D}_{1} h\left(y_{1}, a\right)=\mathrm{D} h_{1}\left(y_{1}\right) h_{2}(a)$. Now choose $a \in \mathbb{F}$ with $h_{2}(a) \neq 0$. Since $\mathscr{S}_{\mathbb{F}}=\mathscr{S}_{\left(y_{1}, y_{2}\right)}$ for any $\left(y_{1}, y_{2}\right) \in \mathbb{F}^{2}$, the matrix

$$
\mathrm{D} F_{a}\left(y_{1}\right)=A(a)+t \mathrm{D} h_{1}\left(y_{1}\right) h_{2}(a)
$$


and hence $\mathrm{D} h_{1}\left(y_{1}\right)$, too, are of the form

$$
\left(\begin{array}{cccc}
u_{1} & -u_{2} & & -u_{l} \\
u_{2} & u_{1} & \ldots & \\
\vdots & & \ddots & \\
u_{l} & & & u_{1}
\end{array}\right) .
$$

Because the function $h_{1}: \mathbb{F} \rightarrow \mathbb{F}$ only takes real values, we have on the other hand

$$
\mathrm{D} h_{1}\left(y_{1}\right)=\left(\begin{array}{ccc}
* & \ldots & * \\
0 & \ldots & 0 \\
\vdots & \ddots & \vdots \\
0 & \ldots & 0
\end{array}\right)
$$

This implies that $\mathrm{D} h_{1}\left(y_{1}\right)=0$ for any $y_{1} \in \mathbb{F}$. Since $h_{1}$ has compact support, this forces $h_{1}$ to be identically zero, which is a contradiction. This shows that the tangent translation plane $\mathscr{A}_{\left(y_{1}, y_{2}\right)}$ is not classical at some point $\left(y_{1}, y_{2}\right) \in \mathbb{F}^{2}$.

\section{References}

[1] Betten D (1970) Nicht-desarguessche 4-dimensionale Ebenen. Arch Math 21: 100-102

[2] Betten D (1973) 4-dimensionale Translationsebenen mit irreduzibler Kollineationsgruppe. Arch Math 24: 552-560

[3] Bödi R (1997) Smooth stable planes. Results Math 31: 300-321

[4] Bödi R (1998) Collineations of smooth stable planes. Forum Math 10: 751-773

[5] Bödi R (1998) Stabilizers of collineation groups of smooth stable planes. Indag Math N S 9: $477-490$

[6] Breitsprecher S (1971) Projektive Ebenen, die Mannigfaltigkeiten sind. Math Z 121: 157-174

[7] Busemann H (1972) The Geometry of Geodesics, New York: Academic Press

[8] Hähl H (1980) Achtdimensionale lokalkompakte Translationsebenen mit großen kompakten Kollineationsgruppen. Monatsh Math 90: 207-218

[9] Hähl H (1987) Sechzehndimensionale lokalkompakte Translationsebenen mit Spin (7) als Kollineationsgruppen. Arch Math 48: 267-276

[10] Husemoller D (1975) Fibre Bundles. New York: Springer

[11] Knarr N (1995) Translation Planes. Lect Notes Math 1611. Berlin Heidelberg New York: Springer

[12] Kolmogoroff A (1932) Zur Begründung der projektiven Geometrie. Ann Math 33: 175-176

[13] Kuz'min EN (1966) Certain classes of division algebras. Algebra i Logika Sem 5: 57-102

[14] Löwe H (1994) Shear planes. Geom Dedicata 52: 87-104

[15] Löwen R (1976) Vierdimensionale stabile Ebenen. Geom Dedicata 5: 239-294

[16] Löwen R (1983) Topology and dimension of stable planes: On a conjecture of H. Freudenthal. J Reine Angew Math 343: 108-122

[17] Löwen R (1989) Compact spreads and compact translation planes over locally compact fields. J Geom 36: 110-116

[18] Otte J (1992) Differenzierbare Ebenen. PhD Thesis, Univ Kiel, Germany

[19] Otte J (1995) Smooth projective translation planes. Geom Dedicata 58: 203-212

[20] Rinow W (1975) Topologie. Berlin: Deutscher Verlag der Wissenschaften

[21] Salzmann H, Betten D, Grundhöfer T, Hähl H, Löwen R, Stroppel M (1995) Compact Projective Planes. Berlin New York: De Gruyter

Authors' addresses: Richard Bödi and Stefan Immervoll, Mathematisches Institut, Universität Tübingen, Auf der Morgenstelle 10, D-72076 Tübingen, Germany e-mail: richard.boedi@ uni-tuebingen.de, stim @michelangelo.mathematik.uni-tuebingen.de; Harald Löwe, Technische Universität Braunschweig, Institut für Analysis, Pockelsstr. 14, D-38106 Braunschweig, Germany, e-mail: harald@riemann.math.nat.tu-bs.de 